\title{
Application of kriging to motorsport aerodynamic analysis
}

\author{
Matthew Cruickshank ${ }^{1, a}$ and Dr Graham Doig ${ }^{2, b}$ \\ ${ }^{12}$ UNSW School of Mechanical and Manufacturing Engineering, Sydney, NSW, Australia \\ ${ }^{a}$ cruickshank.matthew@gmail.com, bg.doig@unsw.edu.au \\ *Matthew Cruickshank, Cruickshank.matthew@gmail.com, +61409 880432
}

\begin{abstract}
Keywords: Kriging, Motorsport Aerodynamics, CFD, Wind Tunnel, Aerodynamic Correlation
\end{abstract}
\begin{abstract}
Aerodynamic analysis in motorsport is conducted using three methods, computational, scaled experimental and full-scale operational. However, the varying fidelity, different sampling resolutions and unavoidable errors of each technique make valid comparisons between datasets from each method difficult and time consuming. Kriging is a geostatistical method to estimate values within a data field by examining and applying the trends of the dataset. This research examines how such techniques can be used to aid comparison between aerodynamic measurements of a race car. It examines how kriging can be used to transform discrete measurements, of varying fidelity and sampling resolution, into semi-continuous measurements, thus allowing computational results to be compared across a wider range of conditions than initially tested. This work explores how kriging can allow the trends from highly sampled data, such as track running, to be applied to less sampled data, such as CFD to improve computational and overall aerodynamic analysis.
\end{abstract}

\section{Introduction}

In modern motorsport aerodynamics has become a key performance differentiator due to the large impact aerodynamic devices have on overall car performance as highlighted by Toet [1]. This results in a much faster rate of aerodynamic development compared with other areas of the aerospace and motoring industry, with cars often being developed week to week [1]. It also sees a variety of analysis methods being used and thus puts demands on analysis techniques that are not seen in other applications. A major issue for many industries is how to assess performance with various pieces of data that are not sampled at the same resolution or in the same exact conditions.

The importance of CFD, and other aerodynamic analysis methods to motorsport is clearly evident as highlighted in [1]. This work highlights that Reynolds Averaged Navier Stokes (RANS) solvers are commonly used within motorsport for aerodynamic development and to understand the differences between the various testing methods. One CFD run examines a single car geometry and condition, taking a number of hours to produce one solution. Thus it is not efficient to test a wide range of points in CFD. However for each solution it does provide a large amount of detail about the nature of the flow surrounding the vehicle. Ultimately CFD is a method to predict the performance of a component, the real test and end usage of any component in motorsport is on track running. [1] clearly identifies the importance of on-track testing but also highlight the difficulties associated with it. Furthermore the work of Petrone et al [2] demonstrates the large effort required to derive accurate aerodynamic component loads from on track testing alone. The contradictions and complexity of motorsport aerodynamic CFD analysis are clearly evident. It is therefore clear that a method to predict the performance of a CFD model across a wider range of conditions, without explicitly testing those conditions would be highly desirable.

Various pieces of research have proposed that kriging and cokriging could fulfill such requirements. Kriging was developed in the 1950s for applications in geostastistics and mining as outlined in Krige's $1951 \mathrm{PhD}$ thesis, [3]. In this work Krige outlines that any estimated point $\left(\widehat{V}\left(x_{0}\right)\right)$, can be considered as a linear combination of known values in a dataset $\left(V\left(x_{n}\right)\right)$, as per,

$$
\widehat{V}\left(x_{0}\right)=\sum_{n=1}^{i} w_{n} V\left(x_{n}\right)
$$


Furthermore the weightings, $w$, for this linear combination can be calculated by examining the variances within the dataset, and considering the location of the estimated point to the known values of the dataset. The work of Matheron, [4] gave further mathematical definitions to the concepts outlined in [3]. There is, therefore, a well-founded theoretical basis for kriging. From this theoretical basis kriging has been extensively applied in a number of other areas. Watson [5] investigated the application of kriging and cokriging to aerodynamic wake measurements. The work of [5] clearly demonstrates that kriging and cokriging can be applied to compare and correlate computational and experimental aerodynamic results. [5] also outlined how kriging can be used to provide quantitative measurements for correlation of aerodynamic measurements in an area that was previously only qualitative.

Laurenceau's 2008 work [6] demonstrates that with all methods of kriging, average errors began to level off when a sampling size greater than 50 is used. Crowel et al [7] confirms this and compares kriging and proper orthogonal decomposition (POD) to interpolate CFD results. The work of [7] details that kriging is, on average, more accurate than POD, however it is less computationally efficient.

Cokriging extends basic kriging to produce a second set of weightings using a second, related dataset however also adds complexity to the analysis. The work of Han et al [8] proposes a new method for cokriging, aiming to reduce its complexity. This work utilises low fidelity CFD results, cokriged with less sampled but higher fidelity CFD results to improve aerodynamic measurements. This work was furthered by Han et al in [9] where hierarchical kriging was utilisied. By applying kriging multiple times building up in fidelity it is possible to produce estimation as accurate as cokriging with reduced resources. These works justify the need for a less complex, more efficient method to compare datasets of varying fidelity. Furthermore the variety of aerodynamic applications of kriging seen in [5], [6], [7], [8] and [9] demonstrate that kriging has the fundamental accuracy to be applied to a range of aerodynamic analysis problems.

\section{Methodology}

Existing wind tunnel (WT) data was provided by Carlin Motorsport that sampled the car at various front and rear rideheights, producing a number of aerodynamic measurements. Further to this a CFD model of the F312 racecar was constructed using geometry supplied by Carlin. Previous works, ([10],[11],[12]) have shown that the K- $\epsilon$ Realizable turbulence model sufficiently predicts the performance inverted wings in ground effect, as such this turbulence model has been applied to the CFD in this work. The flow was assumed to be incompressible as [12] and [13] show this assumption is within the bounds of accuracy for this study. The added accuracy of compressible flow modelling seen in these works did not outweigh reduction in the number of test cases caused by the added resources that would be required. An unstructured mesh, constructed in BETA-CAE ANSA was used and the solution solved using Fluent. In total the mesh comprised of approximately 20 million cells. A $\mathrm{y}^{+}>30$ has been used for all CFD cases due to the complex geometry of the F312 and the accurate performance of the K- $\epsilon$ Realizable turbulence model at this $\mathrm{y}^{+}$[14]. The CFD model was validated against existing WT data. For a full WT replication geometry results were found to be within $2.5 \%$ and $4.8 \%$ for lift and drag respectively. All solutions were completed on UNSW's high performance Trentino cluster across 64 processes.

In addition to the WT data, track data was also supplied by Carlin Motorsport. As outlined above modelling the precise aerodynamic loads from on track data is extremely difficult, however with some simple assumptions aerodynamic trends can be easily extracted from suspension deflection readings, as per Nowlan [15]. For simplification and comparison to the WT and CFD results only straight-line running has been considered. In this running the impact of acceleration on suspension readings was minimised by only considering points where acceleration was negligible. Despite this, minor acceleration and vibration will still be present in the data, which partially accounts for the increased variability seen in the track interpolation. When the car is travelling in a straight line it is assumed that the only variable force acting upon the suspension is aerodynamic. Some forces, other than aerodynamic loads, do act on the suspension during on track running. However, these other 
forces are more constant and thus do not vary with front and rear ride height to the extent of the aerodynamic forces. As a result the change in suspension loading with ride height can be considered primarily due to aerodynamics. As such, it can be clearly seen that the net aerodynamic force can be considered approximately equal to the sum of the suspension forces at each of the four springs. As such the following formulas have been used to estimate the aerodynamic loads acting on the F312 in track running,

$$
\begin{aligned}
& F_{\text {susp }}=M R K_{\text {sn }} \delta_{\text {sn }} \\
& F_{\text {aero }}=\sum F_{\text {susp }}
\end{aligned}
$$

Where $M R=$ motion ratio, which relates the amount of wheel movement to the deflection of the suspension as determined by suspension geometry, $K=$ suspension spring rate and $\delta$ is the deflection of the suspension in $\mathrm{mm}$.

Kriging has been used to examine the change in aerodynamic loading with front and rear ride height. The advantage of kriging over other interpolation methods is that it first examines the dataset to determine the trends and then uses this to inform its interpolation, rather than relying on a generic interpolation function, such as a basic polynomial. The first step is to examine the covariance in the dataset, by considering the distances between all known points in a dataset a variogram can be fitted that models the covariance in the dataset at distance $h$. For this work a tuned Gaussian variogram has been utilised, as per:

$$
\gamma_{\text {Gaus }}=1-e^{-3(h \beta)^{2}}
$$

In this variogram, $\beta$, has been used to tune the variogram to fit the dataset with $h$ being the separation distance between the data points. Kriging aims to minimise the variance of the theoretical error of the estimated point $\left(x_{o}\right)$, by differentiating the variance expression the following system of equation is produced which Isaaks and Srivastava [16] refer to as the ordinary kriging system.

$$
C w=D
$$

Where $C$ is the modelled variogram of the known points in the dataset, $w$ is the weightings to be applied to each datapoint and $D$ is the modelled variogram of the estimated point to the known points. For brevity the full derivation will not be discussed but can be found in numerous resources such as [16]. Through this it is possible to produce aerodynamic measurements across a wider range of conditions than initially explicitly tested. It can be seen that the ordinary kriging system is a function of both the variogram, $\left(\gamma_{n}\right)$, and the dataset, $\left(\mathbb{R}_{n}\right)$, with $\gamma_{n}$ being used to calculate $C$ and $D$ for the various measured values in $\mathbb{R}_{n}$. Therefore the trends, of various datasets $\left(\gamma_{1}, \gamma_{2} \ldots\right)$ can be applied to each discrete dataset, $\mathbb{R}_{n}$. Each dataset $\left(\mathbb{R}_{n}\right)$ will, however, have its own natural level of total covariance. This is primarily influenced by the noise and drift within the data, rather than the actual trend of the dataset. To account for this each variogram, $\left(\gamma_{n}\right)$, has been normalised by total covariance. This ensures that all variograms reach unity, rather than total covariance, as $h$ increases. This process ensures that each variogram can be scaled to match the total covariance of the dataset it is to be applied to, allowing variograms from each method to be applied to any other methods dataset. This is akin to cokriging; however, this simple procedure negates the complexity of cokriging. This process requires the same level of resources as basic kriging however results in an outcome similar to that of cokriging.

\section{Results and Discussion}

In this work nine CFD test points have been considered. In contrast to the CFD measurements the track data took in approximately 200 straight line measurements during the one lap considered. The wider range of sampling points provided by the WT and track allow for a more accurate variogram to be fitted to the datasets. As can be seen in Fig. 1 that the track dataset has a slightly different distribution, to the other datasets, with unity reached at a much higher $h$. All three datasets 
do, however, show Gaussian distribution as seen in Fig. 1. The on track ride height measurements will have a margin of error and noise attached to them. This, in combination with the wider range of sampled points results in the slightly different distribution. The impact of these errors, however, has been minimised by filtering the data before analysing it, in line with method discussed in [2]. However, one must acknowledge and consider the variability and range of on track measurements as this is what the car will experience in real life.

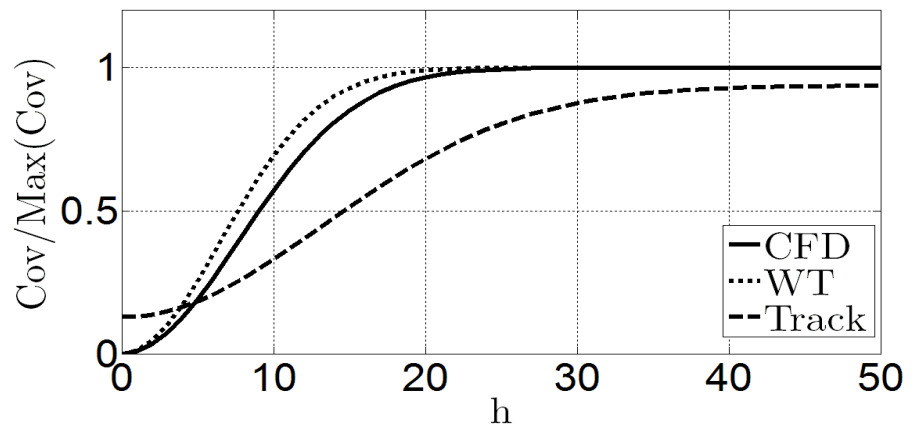

Figure 1-Normalised Variograms comparison

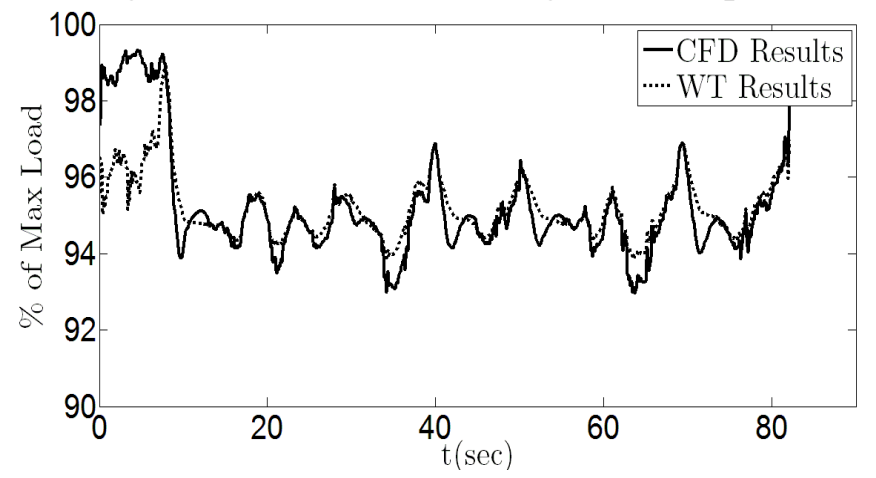

Figure 2- CFD and WT results interpolated over the track ride height conditions

This work has focused on the predicted load values from the WT and CFD datasets. It has used the raw datasets and a kriging procedure to produce a prediction of load over the range of ride heights experienced during one lap of on track running for each method of analysis, as seen in Fig. 2 . The range of ride heights seen in the track lap sees $h$ vary by approximately $30 \mathrm{~mm}$ over the interpolation. Both WT and CFD were sampled at the same velocity and as such the interpolation has only considered the impact of front and rear ride height on load. The pure act of interpolating the two datasets across the wider range of track front and rear ride heights demonstrates the value of the kriging process for aerodynamic analysis. The kriging process utilisied extends the CFD and WT results across a much wider range of conditions, using the discrete raw results as a basis.

To simulate all 800 track front and rear ride height measurements in both CFD and WT would take considerable resources and in many cases is not possible. The kriging process used reduces the time and resources required to estimate the CFD results over various conditions. While the process may not produce results of perfect accuracy it does given a valuable insight into an area which otherwise lacked detail. It can be clearly seen in Fig. 2 that the CFD results, do in general, agree with the WT results. However at several key areas they deviate. It was known that the areas of deviation all occur at a somewhat similar front and rear ride height combination. Further investigation at these conditions could thus provide vital information about the limitations of the CFD model and guide future development of the computational model. 


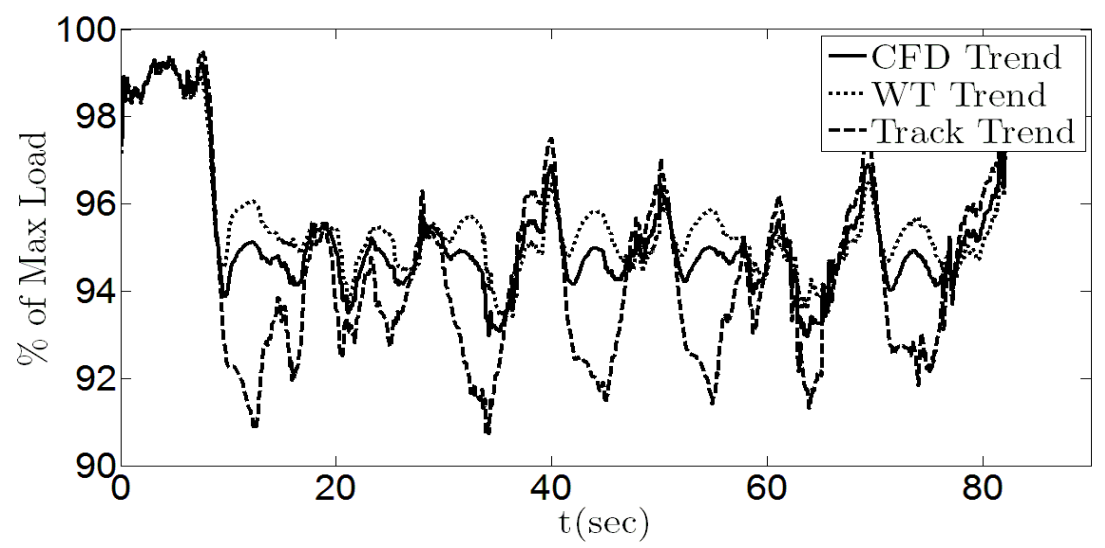

Figure 3- Comparison of interpolated CFD results using trends of various datasets

The process outlined previously allows for the trends seen in the various datasets to be applied to the distinct CFD measurements. The variograms, and thus trends, from the three analysis methods have been applied to the nine CFD readings and interpolated over the timed lap, as seen in Fig. 3. The three interpolations have used the same base dataset, with only the difference in the kriging process being the variogram applied. As a result the differences seen in Fig. 3 represent the differences in the trends predicted by the three methods. The higher variability of the track data is clearly seen. This variability is partially due to influences from variables, such as car vibration, that cannot be controlled at the level seen in the WT and CFD and the inherent noise of track measurements as highlighted by [1] and [2] and discussed previously in regards to the track variogram. Furthermore when considered with Fig. 2 the process has highlighted both the ride height conditions where the CFD results differ from the WT and the areas where the trends of the CFD results differ. By extending the CFD results to a wider range of test points than initially individually sampled in CFD, large amounts of detail regarding the CFD results, has been provided that would otherwise not be evident if only the raw results were used.

\section{Summary}

The kriging process developed extends the initial CFD results to a wider range of testing conditions and gives added detail regarding the CFD results that would not be possible if only raw results were considered. This substantially reduces the time and effort required to produce CFD results across a wide range of testing points. The value of this low resource method can be clearly seen, especially when one considers the time and resources required to obtain similar results over the 800 track test points using CFD testing at each point. This process also uses the discrete measurements of CFD, and applies the other methods variograms to the interpolation, providing further detail on the CFD model that would not be possible otherwise. Although this work has focused on motorsport analysis it is believed that the results of this work could be readily applied to a variety of computational analysis.

\section{Acknowledgements}

I would like to thank David Brown and Carlin Motorsport for supporting this project in a variety of methods, from supplying data and CAD parts to general advice. I would also like to thank BETA-CAE who were generous enough to provide the meshing software free of charge. 


\section{References}

[1] W. Toet, "Aerodynamics and aerodynamic research in Formula 1," Aeronautical Journal, vol. 117, pp. 1-26, 2013.

[2] N. Petrone, M. Capuzzo, E. De Paoli, and N. Biliato. (2002). Acquisition and Analysis of Aerodynamic Loads on Formula 3 Racing Car Wings using Dynamometric Load Cells.

[3] D. G. Krige, "A statistical approach to some mine valuation and allied problems on the Witwatersrand," University of the Witwatersrand, 1951.

[4] G. Matheron, Les variables régionalisées et leur estimation: Paris, 1965.

[5] I. Watson, "Spatial Verification and Validation of Datasets in Fluid Dynamics," PhD, School of Mechanical and Manufacturing Engineering, University of New South Wales, Sydney Australia, 2009.

[6] J. Laurenceau and P. Sagaut, "Building Efficient Response Surfaces of Aerodynamic Functions with Kriging and Cokriging," AIAA Journal, vol. 46, pp. 498-507, 2008.

[7] A. R. Crowell and J. J. McNamara, "Model Reduction of Computational Aerothermodynamics for Hypersonic Aerothermoelasticity," AIAA Journal, vol. 50, pp. 74-84, 2012.

[8] Z. Han, R. Zimmerman, and S. Görtz, "Alternative Cokriging Method for Variable-Fidelity Surrogate Modeling," AIAA Journal, vol. 50, pp. 1205-1210, 2012.

[9] Z.-H. Han and S. Görtz, "Hierarchical Kriging Model for Variable-Fidelity Surrogate Modeling," AIAA Journal, vol. 50, pp. 1885-1896, 2012.

[10] G. Doig and T. J. Barber, "Considerations for Numerical Modeling of Inverted Wings in Ground Effect," AIAA Journal, vol. 49, pp. 2330-2333, 2011.

[11] S. Diasinos, "The aerodynamic interection of a rotating wheel and downforce producing wing in ground effect," School of Mechanical and Manufacturing Engineering, University of New South Wales, Sydney, Australia, 2009.

[12] J. Keogh, "Compressibility Effects Around an Open-Wheeled Race-Car," B.Eng, School of Mechanical and Manufacturing Engineering, University of New South Wales, Sydney Australia, 2012.

[13] G. Doig, T. J. Barber, and A. J. Neely, "The influence of compressibility on the aerodynamics of an inverted wing in ground effect," Journal of fluids engineering, vol. 133, 2011.

[14] Fluent 12.0 User Guide: ANSYS Inc, 2009.

[15] D. Nowlan, "Refining aeromaps from pitch data," Racecar Engineering, vol. March, 2013.

[16] E. H. Isaaks and R. M. Srivastava, Applied geostatistics: Oxford University Press, 1989. 